$7 \quad{ }^{1}$ Meghan E. Vidt, ${ }^{2,3}$ Anthony C. Santago II, ${ }^{4}$ Anthony P. Marsh, ${ }^{5}$ Eric J. Hegedus, ${ }^{6}$ Christopher J.
Short Communication

Ms. No. BM-D-15-00957R1, Rev. 2, Revised Copy

\section{The effects of a rotator cuff tear on activities of daily living in older adults: a kinematic analysis}

Tuohy, ${ }^{6}$ Gary G. Poehling, ${ }^{6}$ Michael T. Freehill, ${ }^{7}$ Michael E. Miller, ${ }^{3}$ Katherine R. Saul

列

.

1. Exercise Science and Health Promotion, Arizona State University, Phoenix,

AZ, USA

2. Virginia Tech-Wake Forest University School of Biomedical Engineering and Sciences, Winston-Salem, NC, USA

3. Department of Mechanical and Aerospace Engineering, North Carolina State

$$
\text { University, Raleigh, NC, USA }
$$

4. Department of Health and Exercise Science, Wake Forest University,

$$
\text { Winston-Salem, NC, USA }
$$

5. Department of Physical Therapy, High Point University, High Point, NC,

\section{USA}

6. Department of Orthopaedic Surgery, Wake Forest School of Medicine,

$$
\text { Winston-Salem, NC, USA }
$$

7. Department of Biostatistical Sciences, Wake Forest School of Medicine,

$$
\text { Winston-Salem, NC, USA }
$$

\title{
1
}

(C) 2016. This manuscript version is made available under the Elsevier user license http://www.elsevier.com/open-access/userlicense/1.0/ 
Corresponding Author: Meghan Vidt, Exercise Science and Health Promotion, Arizona

26 State University, 550 North $3^{\text {rd }}$ Street, Phoenix, AZ85004; Phone: 602-827-2280; Fax:

27 602-827-2253; Email:

28

29 Keywords: upper limb; thoracohumeral; kinematics; aging; rotator cuff tear

30

$31 \quad$ Word Count (Introduction - Discussion): 1,999

32 Word Count (Introduction - Acknowledgements): 2,178 


\section{Abstract}

34 Rotator cuff tears (RCT) in older individuals may compound age-associated

35 physiological changes and impact their ability to perform daily functional tasks. Our objective

36 was to quantify thoracohumeral kinematics for functional tasks in 18 older adults (mean

37 age=63.3 \pm 2.2 ), and compare findings from nine with a RCT to nine matched controls. Motion

38 capture was used to record kinematics for 7 tasks (axilla wash, forward reach, functional pull,

39 hair comb, perineal care, upward reach to $90^{\circ}$, upward reach to $105^{\circ}$ ) spanning the upper limb

40 workspace. Maximum and minimum joint angles and motion excursion for the three

41 thoracohumeral degrees of freedom (elevation plane, elevation, axial rotation) were identified for

42 each task and compared between groups. The RCT group used greater minimum elevation angles

43 for axilla wash and functional pull $(\mathrm{p} \leq 0.0124)$ and a smaller motion excursion for functional pull

44 ( $\mathrm{p}=0.0032)$ compared to the control group. The RCT group also used a more internally rotated

45 maximum axial rotation angle than controls for functional reach, functional pull, hair comb, and

46 upward reach to $105^{\circ}(\mathrm{p} \leq 0.0494)$. The most differences between groups were observed for axial

47 rotation, with the RCT group using greater internal rotation to complete functional tasks, and

48 significant differences between groups were identified for all three thoracohumeral degrees of

49 freedom for functional pull. We conclude that older adults with RCT used more internal rotation

50 to perform functional tasks than controls. The kinematic differences identified in this study may

51 have consequences for progression of shoulder damage and further functional impairment in

52 older adults with RCT.

54 Clinical Trials Registration Number: NCT\#01459536 
57 Sarcopenia and reduced strength are well-known sequelae of aging contributing to

58 functional declines older adults experience (Clark and Manini, 2010). Presence of a shoulder

59 injury, like a rotator cuff tear (RCT), can further reduce an individual's ability to perform

60 functional tasks (Lin et al., 2008; van Schaardenburg et al., 1994). RCT is a common

61 musculoskeletal injury for older adults, with prevalence increasing from $25.6 \%$ to $50.0 \%$ for

62 adults in their sixties and eighties, respectively (Yamamoto et al., 2010). Shoulder injury may

63 result in adaptive movements caused by muscle weakness or force imbalance (Lippitt and

64 Matsen, 1993; Lippitt et al., 1993; Magarey and Jones, 2003; Phadke et al., 2009), or used as a

65 pain avoidance strategy (Hall et al., 2011; Mell et al., 2005). In addition to limiting functionality,

66 altered kinematics may expose the glenohumeral joint to new contact force scenarios, which

67 could lead to further joint damage (Hsu et al., 2003; Vidt, 2014).

68 The upper limb is critical for daily functional tasks, including eating and personal

69 hygiene (Katz et al., 1963). Studies evaluating upper limb functional task performance have

70 primarily focused on younger or uninjured individuals (Magermans et al., 2005; Safaee-Rad et

71 al., 1990; van Andel et al., 2008). Little work has focused on older adults (Hall et al., 2011) or

72 investigated functional task performance in those with RCT. Therefore, our objective was to

73 quantify thoracohumeral kinematics for a group of older adults with and without RCT during

74 performance of functional tasks spanning the upper limb workspace. Kinematics for three

75 thoracohumeral degrees of freedom were compared between groups. The null hypothesis was

76 that task kinematics for RCT and control groups would not be different. 
Eighteen older individuals (mean age $=63.3 \pm 2.2$ ) participated (Table 1); 9 participants

81 (4F/5M) had RCT of the supraspinatus tendon; 9 were age- and sex-matched controls. RCT

82 participants had an MRI-confirmed supraspinatus tendon tear ( $\geq 50 \%$ tendon thickness) and were

83 recruited from our institution's orthopaedic clinic, where they sought treatment for RCT

84 symptoms. Controls were recruited from the local community, had no history of shoulder pain or

85 injury, and were screened for shoulder pain and weakness using a modified lateral Jobe's test

86 (Gillooly et al., 2010), whereby manual resistance was applied to arms elevated $90^{\circ}$ in the

87 scapular plane with neutral axial rotation. RCT participants' injured arm was studied and the

88 dominant arm was investigated for controls. Wake Forest Health Sciences Institutional Review

89 Board approved this study; all participants provided written informed consent prior to

90 participation.

\section{$92 \quad$ Functional tasks}

93 Participants completed 7 functional tasks spanning the upper limb workspace: forward

94 reach, functional pull, upward reach to a shelf at shoulder height (upward reach $90^{\circ}$ ) and $15^{\circ}$

95 above horizontal (upward reach $105^{\circ}$ ), axilla wash, perineal care, and hair comb. All tasks were

96 completed while seated (chair height $=0.53 \mathrm{~m}$ ) at a table (height $=0.775 \mathrm{~m}$ ). Descriptions of each

97 task and associated loads, selected to mimic typical loads in daily performance, are described in

98 Table 2. Participants were given instructions on start and finish hand positions for each task, but

99 could freely choose their joint postures and speed during each movement. Task order was

100 randomized; three trials of each task were recorded before proceeding to the next task.

101 Participants were provided 60sec rest between trials and $2 \mathrm{~min}$ rest between tasks. Participants 
102 were instructed to stop a task if they experienced any pain or discomfort (see below). The second 103 trial of each task for each participant was used for analysis.

104 Positions of twelve $1 \mathrm{~cm}$ retro-reflective markers placed on the upper limb and torso

105 (Figure 1) were tracked at $200 \mathrm{~Hz}(60 \mathrm{~Hz}$ for 3 participants) using 7 Hawk motion capture 106 cameras (Motion Analysis Corporation, Santa Rosa, CA). Marker data was post-processed and 107 smoothed with a 6Hz Butterworth filter using Cortex software (Motion Analysis Corporation). 108

\section{$109 \quad$ Kinematic calculations}

110 A dynamic upper limb model (Saul et al., 2015) was implemented in OpenSim (v.3.1)

111 (Delp et al., 2007). The model was scaled to each participant using marker locations from one 112 static trial. Following scaling, the inverse kinematics tool calculated joint kinematics for each 113 task. Kinematic trajectories were filtered with a zero-phase filter using a custom Matlab program 114 (The Mathworks, Natick, MA). Consistent with ISB standards describing thoracohumeral motion 115 (Wu et al., 2005), joint angles were decomposed by applying Y-X-Y rotation order, 116 corresponding to elevation plane, elevation, and axial rotation, using axes defined from 117 anatomical landmarks. Elevation in $0^{\circ}$ elevation plane is abduction; elevation in $90^{\circ}$ elevation 118 plane is forward flexion; positive axial rotation is internal rotation; negative axial rotation is 119 external rotation. Maximum and minimum angles were calculated for each degree of freedom 120 (Figure 1). Motion excursion was calculated by subtracting the minimum angle from the 121 maximum angle. To compare across participants, kinematics were normalized by task 122 completion time and are presented as a percentage of total movement time.

\section{$124 \quad$ Statistical analysis}


126 for hand dominance, was used to separately evaluate differences between RCT and control

127 groups for maximum angle, minimum angle, and motion excursion of each degree of freedom for

128 each task (v.9.3, SAS Institute, Inc., Cary, NC). Significance was set at $\mathrm{p} \leq 0.05$. We did not 129 adjust for type I error due to the exploratory nature of these analyses.

\section{$131 \quad$ Results}

Six RCT participants had full-thickness supraspinatus tear. Tears extended into

133 infraspinatus in 7 participants and subscapularis in 5 participants. Three RCT participants did not

134 complete all tasks due to pain: one could not complete perineal care and hair comb; one could 135 not complete hair comb; one could not complete upward reach $105^{\circ}$. RCT participants completed 136 several tasks with different kinematics than controls (Figure 2; Supplement 1, 2). Elevation plane

137 was similar between groups for most tasks, but RCT participants used a smaller (closer to 138 abduction plane) maximum elevation plane for forward reach $(\mathrm{p}=0.0300)$ and functional pull $139(\mathrm{p}=0.0020)$ (Figure 2; Table 3). Minimum elevation angle for RCT participants was greater 140 (more elevated) for axilla wash $(\mathrm{p}=0.0124)$ and functional pull $(\mathrm{p}=0.0047)$. RCT participants had 141 smaller motion excursion for elevation during functional pull $(\mathrm{p}=0.0032)$. RCT participants used 142 greater maximum internal rotation angles for forward reach $(\mathrm{p}=0.0004)$, functional pull $143(\mathrm{p}=0.0296)$, hair comb $(\mathrm{p}=0.0494)$, and upward reach $105^{\circ}(\mathrm{p}=0.0337)$ than controls, and greater 144 minimum internal rotation during upward reach $105^{\circ}(\mathrm{p}=0.0089)$ compared to controls.

\section{Discussion}

147 We found older individuals with RCT completed functional reach, functional pull, hair 148 comb, and upward reach $105^{\circ}$ with more internal rotation than controls. The RCT group 
149 completed functional pull most differently, with differences identified in all three degrees of

150 freedom. Kinematic differences identified for RCT participants across tasks may precipitate

151 glenohumeral joint damage and increase functional impairment.

152 Three RCT participants could not complete all tasks due to pain because of extreme

153 motion excursion requirements for hair comb and upward reach $105^{\circ}$. Pain observed in these

154 participants is consistent with elevation and external rotation pain assessed clinically with

155 physical (e.g. Beaudreuil et al., 2009) and self-report (e.g. MacDermid et al., 2004) assessments.

156 One subject could not perform perineal care because axial rotation and sagittal plane elevation

157 movements were limited by concomitant biceps tendon and superior labral pathology. While our

158 analyses did not detect statistically different movements between groups for these tasks, this may

159 be because failed trials were excluded from analyses. Movements requiring large motion

160 excursion should be targets for adaptive movement strategy development to facilitate

161 independence for older adults (Hall et al., 2011). Identification of individuals in this small cohort

162 who could not perform these tasks suggests many older adults with RCT would be limited in

163 movements requiring relatively large motion excursions in one (e.g. upward reach $105^{\circ}$ ) or more

164 (e.g. hair comb) degrees of freedom.

165 These RCT participants consistently demonstrated greater internal rotation during

166 functional reach, functional pull, hair comb, and upward reach $105^{\circ}$. This suggests internal

167 rotation may be an adaptive strategy, possibly to avoid pain. Seven RCT participants had tears

168 extending into infraspinatus, and external rotation can exacerbate pain when infraspinatus is

169 involved (Beaudreuil et al., 2009). Efforts to avoid painful postures may expose the shoulder to

170 impingement, particularly at more elevated postures (Ellenbecker and Cools, 2010).

171 Alternatively, increased internal rotation may result from muscle force imbalance; strength 
172 assessments for these same RCT participants (Vidt et al., 2015) demonstrated markedly reduced

173 external rotation strength. Force imbalances may affect glenohumeral loading characteristics,

174 which can lead to further joint damage (Hsu et al., 2003) and other pathology, like humeral head

175 translation. Contrasting our findings, Hall et al. (2011) studied functional upper limb tasks and

176 reported older adults with impingement used less internal rotation than controls; these conflicting

177 findings may result from studying different shoulder pathologies, or the high prevalence of 178 concomitant infraspinatus tears in this study's cohort.

179 Increased internal rotation for RCT participants may be due to deltoid and pectoral 180 muscle compensation in the absence of a fully functional rotator cuff. Normally, muscular 181 activation and co-activation strategies provide glenohumeral stability during movement (Hawkes 182 et al., 2012a). Following RCT, other muscles must compensate for damaged rotator cuff 183 muscle(s) to maintain stability (Hawkes et al., 2012b). Kinematic differences observed here, 184 particularly for internal rotation, may reflect altered muscle activation patterns following RCT 185 (Hawkes et al., 2012b), or age-associated altered co-contraction (Klein et al., 2001). Future work 186 should explore relationships between muscle activation and functional task kinematics, including 187 tasks requiring large motion excursion (e.g. hair comb) and load movement (e.g. forward reach) 188 for individuals with RCT. Such work would provide insight into which treatment paradigms 189 could best improve functional performance or aid in adaptive strategy development promoting 190 functional independence.

191 Kinematic differences identified following RCT may expose the shoulder to increased 192 risk for subsequent damage and functional impairment. For example, greater internal rotation 193 observed for RCT participants may indicate subacromial impingement, which reduces the space 194 between the humeral head and acromion, compressing the subacromial bursa and rotator cuff 
195 tendons (Braman et al., 2014; Michener et al., 2003). Previous work reports glenohumeral 196 internal rotation is associated with increased risk for impingement of anterior structures (Flatow 197 et al., 1994; Ludewig and Braman, 2011; Werner et al., 2006; Yanai et al., 2006). The underlying 198 cause of RCT is likely multifactorial, and additional factors, like overuse, overhead activities, or 199 acromial anatomy, may further contribute to RCT development (Soslowsky et al., 2002). While 200 it remains unknown whether impingement causes or results from RCT (Braman et al., 2014; 201 Ludewig and Braman, 2011; Michener et al., 2003), RCT-associated impingement can contribute 202 to further RCT progression (Ludewig and Braman, 2011), possibly exacerbating functional 203 limitations. Restoration of internal rotation during functional tasks could reduce impingement 204 risk, but pain associated with concomitant infraspinatus tearing may preclude use of externally 205 rotated postures.

206 This work has limitations. A small cohort of 18 individuals was studied. Our sample 207 included individuals with full- and partial-thickness supraspinatus tears, and included 208 participants with tears extending into infraspinatus or subscapularis tendons, although none were 209 full-width. However, all patients experienced RCT symptoms, sought treatment, and had tear 210 confirmation with MRI. Controls were screened using a lateral Jobe's test. Sensitivity of this 211 manual exam is $81 \%$ (Gillooly et al. 2010), thus it is possible that an asymptomatic tear was not 212 identified. Controls did not receive an MRI to screen for asymptomatic RCT, but since $16.9 \%$ of 213 the population has been reported to have an asymptomatic RCT (Yamamoto et al., 2010), future 214 studies should use MRI to screen for asymptomatic tears. This study evaluated thoracohumeral 215 kinematics but did not examine scapulothoracic or glenohumeral kinematics. Since all joints of 216 the shoulder girdle contribute to upper limb movement, future work should also examine a 217 RCT's effect on scapulothoracic and glenohumeral kinematics. The significant differences in 
218 kinematics identified here motivate further exploration of these and additional functional tasks in

219 a larger cohort to more comprehensively characterize functional implications of RCT in older 220 adults.

221 This study provides baseline measures of thoracohumeral kinematics associated with 222 several common functional tasks in older adults with and without RCT, which can be compared 223 to other pathologies or groups following treatment. Additionally, these data may be used in 224 conjunction with computational analysis (e.g. Vidt, 2014) to further explore strength 225 requirements, joint loading, and clinical implications of RCT-induced alterations to upper limb 226 kinematics.

\section{Acknowledgements}

229 This study was funded by the National Institute on Aging of the National Institutes of Health 230 (\#F31AG040921 (Vidt)), the Wake Forest University Claude D. Pepper Older Americans 231 Independence Center (\#P30AG021332), the National Science Foundation (\#1405246 (Saul)), the 232 Wake Forest Center for Biomolecular Imaging and the Wake Forest School of Medicine 233 Translational Science Institute Clinical Research Unit. The sponsors of this study had no 234 involvement in study design, collection, analysis, or interpretation of data, writing of this 235 manuscript, or the decision to submit to this journal for publication.

238 Beaudreuil, J., Nizard, R., Thomas, T., Peyre, M., Liotard, J.P., Boileau, P., Marc, T., Dromard, 239 C., Steyer, E., Bardin, T., Orcel, P., Walch, G., 2009. Contribution of clinical tests to the 240 diagnosis of rotator cuff disease: a systematic literature review. Joint Bone Spine 76 (1), $15-19$. 
Braman, J.P., Zhao, K.D., Lawrence, R.L., Harrison, A.K., Ludewig, P.M., 2014. Shoulder impingement revisited: evolution of diagnostic understanding in orthopedic surgery and physical therapy. Med. Biol. Eng. Comput. 52 (3), 211-219.

Clark, B.C., Manini, T.M., 2010. Functional consequences of sarcopenia and dynapenia in the elderly. Curr. Opin. Clin. Nutr. Metab. Care 13 (3), 271-276.

Delp, S.L., Anderson, F.C., Arnold, A.S., Loan, P., Habib, A., John, C.T., Guendelman, E., Thelen, D.G., 2007. OpenSim: open-source software to create and analyze dynamic simulations of movement. IEEE Trans. Biomed. Eng. 54 (11), 1940-1950.

Ellenbecker, T.S., Cools, A., 2010. Rehabilitation of shoulder impingement syndrome and rotator cuff injuries: an evicence-based review. Br. J. Sports Med. 44, 319-327.

Flatow, E.L., Soslowsky, L.J., Ticker, J.B., Pawluk, R.J., Hepler, M., Ark, J., Mow, V.C., Bigliani, L.U., 1994. Excursion of the rotator cuff under the acromion. Patterns of subacromial contact. Am. J. Sports Med. 22 (6), 779-788.

Gillooly, J.J., Chidambaram, R., Mok, D., 2010. The lateral Jobe test: A more reliable method of diagnosing rotator cuff tears. Int. J. Shoulder Surg. 4 (2), 41-43.

Hall, L.C., Middlebrook, E.E., Dickerson, C.R., 2011. Analysis of the influence of rotator cuff impingements on upper limb kinematics in an elderly population during activities of daily living. Clin. Biomech. 26 (6), 579-584.

Hawkes, D.H., Alizadehkhaiyat, O., Fisher, A.C., Kemp, G.J., Roebuck, M.M., Frostick, S.P., 2012a. Normal shoulder muscular activation and co-ordination during a shoulder elevation task based on activities of daily living: an electromyographic study. J. Orthop. Res. 30 (1), 53-60. 
Hawkes, D.H., Alizadehkhaiyat, O., Kemp, G.J., Fisher, A.C., Roebuck, M.M., Frostick, S.P., 2012b. Shoulder muscle activation and coordination in patients with a massive rotator cuff tear: an electromyographic study. J. Orthop. Res. 30 (7), 1140-1146.

267

268

269

270

271

272

273

274

275

276

277

278

279

280

281

282

283

284

285

Hsu, H.C., Luo, Z.P., Stone, J.J., Huang, T.H., An, K.N., 2003. Correlation between rotator cuff tear and glenohumeral degeneration. Acta Orthop. Scand. 74 (1), 89-94.

Katz, S., Ford, A.B., Moskowitz, R.W., Jackson, B.A., Jaffe, M.W., 1963. Studies of Illness in the Aged. The Index of Adl: A Standardized Measure of Biological and Psychosocial Function. JAMA 185, 914-919.

Klein, C.S., Rice, C.L., Marsh, G.D., 2001. Normalized force, activation, and coactivation in the arm muscles of young and old men. J. Appl. Physiol. 91 (3), 1341-1349.

Lin, J.C., Weintraub, N., Aragaki, D.R., 2008. Nonsurgical treatment for rotator cuff injury in the elderly. J. Am. Med. Dir. Assoc. 9 (9), 626-632.

Lippitt, S., Matsen, F., 1993. Mechanisms of glenohumeral joint stability. Clin. Orthop. Relat. Res. 291, 20-28.

Lippitt, S.B., Vanderhooft, J.E., Harris, S.L., Sidles, J.A., Harryman, D.T., 2nd, Matsen, F.A., 3rd, 1993. Glenohumeral stability from concavity-compression: A quantitative analysis. J. Shoulder Elbow Surg. 2 (1), 27-35.

Ludewig, P.M., Braman, J.P., 2011. Shoulder impingement: biomechanical considerations in rehabilitation. Man. Ther. 16 (1), 33-39.

MacDermid, J.C., Ramos, J., Drosdowech, D., Faber, K., Patterson, S., 2004. The impact of rotator cuff pathology on isometric and isokinetic strength, function, and quality of life. J. Shoulder Elbow Surg. 13 (6), 593-598. 
Magarey, M.E., Jones, M.A., 2003. Specific evaluation of the function of force couples relevant for stabilization of the glenohumeral joint. Man. Ther. 8 (4), 247-253.

Magermans, D.J., Chadwick, E.K., Veeger, H.E., van der Helm, F.C., 2005. Requirements for upper extremity motions during activities of daily living. Clin. Biomech. 20 (6), 591-599.

Mell, A.G., LaScalza, S., Guffey, P., Ray, J., Maciejewski, M., Carpenter, J.E., Hughes, R.E., 2005. Effect of rotator cuff pathology on shoulder rhythm. J. Shoulder Elbow Surg. 14 (1

Michener, L.A., McClure, P.W., Karduna, A.R., 2003. Anatomical and biomechanical mechanisms of subacromial impingement syndrome. Clin. Biomech. 18 (5), 369-379.

Phadke, V., Camargo, P., Ludewig, P., 2009. Scapular and rotator cuff muscle activity during arm elevation: A review of normal function and alterations with shoulder impingement. Rev. Bras. Fisioter. 13 (1), 1-9.

Safaee-Rad, R., Shwedyk, E., Quanbury, A.O., Cooper, J.E., 1990. Normal functional range of motion of upper limb joints during performance of three feeding activities. Arch. Phys. Med. Rehabil. 71 (7), 505-509.

Saul, K., Hu, X., Goehler, C., Vidt, M., Daly, M., Velisar, A., Murray, W., 2015. Benchmarking of dynamic simulation predictions in two software platforms using an upper limb musculoskeletal model. Comput. Methods Biomech. Biomed. Eng. 18 (13), 1445-1458.

Soslowsky, L.J., Thomopoulos, S., Esmail, A., Flanagan, C.L., Iannotti, J.P., Williamson, J.D., 3rd, Carpenter, J.E., 2002. Rotator cuff tendinosis in an animal model: role of extrinsic and overuse factors. Ann. Biomed. Eng. 30 (8), 1057-1063. 3D kinematics of upper extremity functional tasks. Gait Posture 27 (1), 120-127. 
van Schaardenburg, D., Van den Brande, K.J., Ligthart, G.J., Breedveld, F.C., Hazes, J.M., 1994. Musculoskeletal disorders and disability in persons aged 85 and over: a community survey. Ann. Rheum. Dis. 53 (12), 807-811.

312 Vidt, M.E., 2014. Muscle structure and function in older adults with a rotator cuff tear. Dissertation, Wake Forest University, Winston-Salem, North Carolina.

314 Vidt, M.E., Santago II, A.S., Tuohy, C.J., Poehling, G.G., Freehill, M.T., Kraft, R.A., Marsh, A.P., Hegedus, E.J., Miller, M.E., Saul, K.R., 2015. Assessments of fatty infiltration and muscle atrophy from a single magnetic resonance image slice are not predictive of threedimensional measurements. Arthroscopy. (Epub ahead of print).

Werner, C.M., Blumenthal, S., Curt, A., Gerber, C., 2006. Subacromial pressures in vivo and effects of selective experimental suprascapular nerve block. J. Shoulder Elbow Surg. 15 (3), 319-323.

Wu, G., van der Helm, F.C., Veeger, H.E., Makhsous, M., Van Roy, P., Anglin, C., Nagels, J., Karduna, A.R., McQuade, K., Wang, X., Werner, F.W., Buchholz, B., 2005. ISB recommendation on definitions of joint coordinate systems of various joints for the reporting of human joint motion--Part II: shoulder, elbow, wrist and hand. J. Biomech. 38 (5), 981-992.

Yamamoto, A., Takagishi, K., Osawa, T., Yanagawa, T., Nakajima, D., Shitara, H., Kobayashi, T., 2010. Prevalence and risk factors of a rotator cuff tear in the general population. J. Shoulder Elbow Surg. 19 (1), 116-120.

Yanai, T., Fuss, F.K., Fukunaga, T., 2006. In vivo measurements of subacromial impingement: substantial compression develops in abduction with large internal rotation. Clin. Biomech. 21 (7), 692-700. 
332 Figure 1: Motion definitions and marker locations. Thoracohumeral motion was defined as

333 elevation plane, elevation, and axial rotation and calculated in accordance with ISB standards

334 (Wu et al., 2005). Elevation in the $0^{\circ}$ elevation plane is abduction and elevation in the $90^{\circ}$

335 elevation plane is forward flexion; positive axial rotation is internal rotation and negative axial

336 rotation is external rotation (Wu et al., 2005). Retro-reflective motion capture markers (red

337 spheres) were placed on anatomical locations, including: the $7^{\text {th }}$ cervical vertebra (C7), the most

338 ventral aspect of the sternoclavicular joint (SC), xiphoid process (XP), the most lateral aspect of

339 the scapular acromial angle (AA), the mid-upper arm (UA), the lateral epicondyle of the humerus

340 (LE), the medial epicondyle of the humerus (ME), the mid-forearm (FA), the styloid process of

341 the radius (RS), the styloid process of the ulna (US), the $2^{\text {nd }}$ metacarpophalangeal joint (2MP),

342 and the $5^{\text {th }}$ metacarpophalangeal joint (5MP). One marker (not shown) was affixed to the top of

343 the dumbbell weight (forward, upward reaches), the weight machine handle (functional pull),

344 and the pencil (hair comb).

345

346 Figure 2: Thoracohumeral kinematics during the performance of planar functional tasks. Mean

$347 \pm$ standard deviation (SD) of kinematics are shown for the rotator cuff tear (RCT) group (gray

348 dashed line, light shaded band) and control group (solid black line, dark shaded band). Mean

349 maximum $( \pm S D)$, mean minimum $( \pm S D)$, and mean motion excursion are shown with bars to the

350 right of the plot, with the horizontal line in the bars representing the mean joint angle for the

351 task. $\$$ indicates significant difference in maximum angle; $\S$ indicates significant difference

352 between groups in minimum angle; * indicates significant difference between groups for motion

353 excursion. For the forward reach task, the RCT group had a smaller (closer to abduction plane)

354 maximal elevation plane $(p=0.0300)$ angle $(A)$ and greater maximum internal rotation angle 
$355(p=0.0004)$ (B) than the control group. For the functional pull, the RCT group used a smaller 356 (closer to abduction plane) maximal elevation plane $(p=0.0020)(C)$, greater minimum elevation 357 angle $(p=0.0047)$, and a smaller elevation motion excursion $(p=0.0032)(D)$, and a greater 358 maximal internal rotation joint angle $(\mathrm{p}=0.0296)(\mathrm{E})$. For the upward reach $105^{\circ}$, the $\mathrm{RCT}$ group 359 used a greater maximum internal rotation angle $(p=0.0337)$ and a greater minimum internal 360 rotation angle $(\mathrm{p}=0.0089)(\mathrm{F})$ than controls. For the axilla wash, the $\mathrm{RCT}$ group used a greater 361 minimum elevation angle $(p=0.0124)(G)$ than controls. For the hair comb, the RCT group used a 362 greater maximum internal rotation angle $(p=0.0494)(H)$ than controls. 
364 Table 3: Group mean differences adjusting for hand dominance for maximum, minimum, and motion

365 excursion (95\% confidence interval) for elevation plane, elevation, and axial rotation for each functional

366 task. * indicates significance.

367

\begin{tabular}{|c|c|c|c|c|c|c|c|}
\hline & Axilla wash & $\begin{array}{c}\text { Forward } \\
\text { reach } \\
\end{array}$ & $\begin{array}{c}\text { Functional } \\
\text { pull }\end{array}$ & Hair comb & $\begin{array}{c}\text { Perineal } \\
\text { care }\end{array}$ & $\begin{array}{c}\text { Upward } \\
\text { reach } 90^{\circ} \\
\end{array}$ & $\begin{array}{c}\text { Upward } \\
\text { reach } 105^{\circ}\end{array}$ \\
\hline \multirow{2}{*}{$\begin{array}{l}\text { Elevation } \\
\text { plane }\end{array}$} & $\begin{array}{c}1.0(-8.3 \\
10.2)\end{array}$ & $\begin{array}{c}9.5(1.1 \\
17.9)\end{array}$ & $\begin{array}{c}13.9(6.0 \\
21.8)\end{array}$ & $\begin{array}{c}2.9(-10.6 \\
16.4)\end{array}$ & $\begin{array}{c}-5.9(-40.6 \\
28.8)\end{array}$ & $\begin{array}{c}3.5(-4.3 \\
11.3)\end{array}$ & $\begin{array}{c}3.5(-4.4 \\
11.3)\end{array}$ \\
\hline & $p=0.8290$ & $p=0.0300 *$ & $p=0.0020^{*}$ & $p=0.6319$ & $p=0.7033$ & $p=0.3473$ & $p=0.3588$ \\
\hline \multirow[t]{2}{*}{ Elevation } & $\begin{array}{c}-7.4(-25.0 \\
10.3)\end{array}$ & $\begin{array}{c}-0.0(-7.9 \\
7.9)\end{array}$ & $0.6(-7.2,8.4)$ & $\begin{array}{c}-3.0(-19.7 \\
13.6)\end{array}$ & $\begin{array}{c}-3.2(-13.5 \\
7.1)\end{array}$ & $\begin{array}{c}-1.1(-8.5 \\
6.2)\end{array}$ & $\begin{array}{c}0.7(-8.3 \\
9.6)\end{array}$ \\
\hline & $p=0.3875$ & $p=0.9974$ & $p=0.8770$ & $p=0.7007$ & $p=0.5153$ & $p=0.7478$ & $p=0.8721$ \\
\hline $\begin{array}{l}\text { Axial } \\
\text { rotation }\end{array}$ & $\begin{array}{c}-9.7(-20.7 \\
1.3) \\
\mathrm{p}=0.0778\end{array}$ & $\begin{array}{c}-13.2(-18.3 \\
-8.0) \\
p=0.0004 *\end{array}$ & $\begin{array}{c}-10.4(-19.6,- \\
1.2) \\
p=0.0296^{*}\end{array}$ & $\begin{array}{c}-13.2(-26.4 \\
-0.0) \\
p=0.0494^{*}\end{array}$ & $\begin{array}{c}-7.5(-30.9 \\
16.0) \\
p=0.4953\end{array}$ & $\begin{array}{c}-4.8(-18.5 \\
8.9) \\
p=0.4664\end{array}$ & $\begin{array}{c}-9.5(-18.1,- \\
1.0) \\
p=0.0337^{*}\end{array}$ \\
\hline \multicolumn{8}{|l|}{ Minimum } \\
\hline & Axilla wash & $\begin{array}{c}\text { Forward } \\
\text { reach }\end{array}$ & $\begin{array}{c}\text { Functional } \\
\text { pull }\end{array}$ & Hair comb & $\begin{array}{c}\text { Perineal } \\
\text { care }\end{array}$ & $\begin{array}{l}\text { Upward } \\
\text { reach } 90^{\circ}\end{array}$ & $\begin{array}{c}\text { Upward } \\
\text { reach } 105^{\circ}\end{array}$ \\
\hline \multirow{2}{*}{$\begin{array}{l}\text { Elevation } \\
\text { plane }\end{array}$} & $\begin{array}{c}-21.0(- \\
63.1,21.0)\end{array}$ & $\begin{array}{c}5.7(-24.7 \\
36.1)\end{array}$ & $\begin{array}{c}5.1(-22.1 \\
32.3)\end{array}$ & $\begin{array}{c}5.5(-26.4 \\
37.3)\end{array}$ & $\begin{array}{c}-3.1(-25.3 \\
19.0)\end{array}$ & $\begin{array}{c}-1.4(-28.1 \\
25.3)\end{array}$ & $\begin{array}{c}11.2(-11.2 \\
33.6)\end{array}$ \\
\hline & $p=0.2902$ & $p=0.6944$ & $p=0.6947$ & $p=0.6975$ & $p=0.7580$ & $p=0.9122$ & $p=0.3005$ \\
\hline \multirow[t]{2}{*}{ Elevation } & $\begin{array}{c}-8.4(-14.7 \\
-2.1)\end{array}$ & $\begin{array}{c}-6.0(-13.3 \\
1.3)\end{array}$ & $\begin{array}{c}-10.0(-16.4,- \\
3.6)\end{array}$ & $\begin{array}{c}-5.5(-15.3, \\
4.2)\end{array}$ & $\begin{array}{c}-3.1(-10.9 \\
4.7)\end{array}$ & $\begin{array}{c}-5.1(-13.7 \\
3.6)\end{array}$ & $\begin{array}{c}-1.1(-6.9 \\
4.7)\end{array}$ \\
\hline & $p=0.0124^{*}$ & $p=0.0992$ & $p=0.0047^{*}$ & $p=0.2289$ & $p=0.4024$ & $p=0.2199$ & $p=0.6696$ \\
\hline $\begin{array}{l}\text { Axial } \\
\text { rotation }\end{array}$ & $\begin{array}{c}-20.8(- \\
42.7,1.1) \\
p=0.0601\end{array}$ & $\begin{array}{c}-6.2(-15.4 \\
3.1) \\
p=0.1674\end{array}$ & $\begin{array}{c}-8.6(- \\
19.0,1.8) \\
p=0.0969\end{array}$ & $\begin{array}{c}-1.5(-28.9 \\
25.9) \\
p=0.9088\end{array}$ & $\begin{array}{c}-21.2(-56.6 \\
14.2) \\
p=0.2140\end{array}$ & $\begin{array}{c}-5.2(-11.3 \\
1.0) \\
p=0.0897\end{array}$ & $\begin{array}{c}-8.7(-14.3,- \\
3.0) \\
p=0.0089 *\end{array}$ \\
\hline \multicolumn{8}{|c|}{ Motion excursion } \\
\hline & Axilla wash & $\begin{array}{c}\text { Forward } \\
\text { reach } \\
\end{array}$ & $\begin{array}{c}\text { Functional } \\
\text { pull }\end{array}$ & Hair comb & $\begin{array}{c}\text { Perineal } \\
\text { care }\end{array}$ & $\begin{array}{c}\text { Upward } \\
\text { reach } 90^{\circ}\end{array}$ & $\begin{array}{c}\text { Upward } \\
\text { reach } 105^{\circ}\end{array}$ \\
\hline \multirow{2}{*}{$\begin{array}{l}\text { Elevation } \\
\text { plane }\end{array}$} & $\begin{array}{c}23.8(-20.1 \\
67.8)\end{array}$ & $\begin{array}{c}4.5(-20.4 \\
29.4)\end{array}$ & $\begin{array}{c}8.8(-16.5 \\
34.1)\end{array}$ & $\begin{array}{c}-3.1(-29.8 \\
23.5)\end{array}$ & $\begin{array}{c}-4.0(-37.6 \\
29.6)\end{array}$ & $\begin{array}{c}4.9(-21.2 \\
31.0)\end{array}$ & $\begin{array}{c}-7.8(-29.7 \\
14.2)\end{array}$ \\
\hline & $p=0.2657$ & $p=0.7061$ & $p=0.4700$ & $p=0.7867$ & $p=0.7964$ & $p=0.6939$ & $p=0.4613$ \\
\hline \multirow[t]{2}{*}{ Elevation } & $\begin{array}{c}1.0(-16.5 \\
18.5)\end{array}$ & $\begin{array}{c}6.2(-0.5 \\
12.8)\end{array}$ & $\begin{array}{c}10.5(4.1 \\
17.0)\end{array}$ & $\begin{array}{c}3.5(-17.3 \\
24.2)\end{array}$ & $\begin{array}{c}-0.0(-11.3 \\
11.2)\end{array}$ & $\begin{array}{c}4.3(-1.6 \\
10.2)\end{array}$ & $\begin{array}{c}2.0(-6.6 \\
10.6)\end{array}$ \\
\hline & $p=0.9038$ & $p=0.0669$ & $p=0.0032 *$ & $p=0.7250$ & $p=0.9951$ & $p=0.1346$ & $p=0.6123$ \\
\hline \multirow{2}{*}{$\begin{array}{l}\text { Axial } \\
\text { rotation }\end{array}$} & $\begin{array}{c}12.7(-7.8 \\
33.3)\end{array}$ & $\begin{array}{c}-6.0(-13.0 \\
1.0)\end{array}$ & $\begin{array}{c}-1.9(-6.8, \\
3.0)\end{array}$ & $\begin{array}{c}-11.8(-43.7 \\
20.1)\end{array}$ & $\begin{array}{c}15.2(-9.5 \\
39.8)\end{array}$ & $\begin{array}{c}0.6(-8.8 \\
10.1)\end{array}$ & $\begin{array}{c}-1.1(-9.1 \\
6.9)\end{array}$ \\
\hline & $p=0.1971$ & $p=0.0880$ & $p=0.4325$ & $p=0.4395$ & $p=0.1991$ & $p=0.8876$ & $p=0.7590$ \\
\hline
\end{tabular}

368 
370 Table 1: Participant characteristics.

\begin{tabular}{|c|c|c|c|c|c|}
\hline Subject & $\begin{array}{c}\text { Age } \\
\text { (years) }\end{array}$ & $\begin{array}{l}\text { Height } \\
\text { (cm) }\end{array}$ & $\begin{array}{c}\text { Body mass } \\
(\mathrm{kg})\end{array}$ & $\begin{array}{l}\text { Dominant } \\
\text { arm }\end{array}$ & $\begin{array}{c}\text { Injured } \\
\text { arm }\end{array}$ \\
\hline RF01 & 65 & 149.9 & 53.5 & Right & Left \\
\hline RF02 & 63 & 160 & 73.5 & Right & Right \\
\hline RF03 & 60 & 180.3 & 122.5 & Right & Right \\
\hline RF04 & 65 & 162.6 & 65.8 & Right & Left \\
\hline RM01 & 61 & 167.6 & 83.9 & Right & Left \\
\hline RM02 & 64 & 177.8 & 108 & Left & Left \\
\hline RM03 & 64 & 182.9 & 88.5 & Right & Left \\
\hline RM04 & 62 & 177.8 & 95.3 & Left & Left \\
\hline RM05 & 66 & 168.9 & 87.1 & Right & Left \\
\hline $\mathrm{CF} 01$ & 67 & 172.7 & 70.8 & Right & N/A \\
\hline $\mathrm{CF} 02$ & 65 & 162.6 & 65.8 & Right & N/A \\
\hline $\mathrm{CF} 03$ & 60 & 157.5 & 79.4 & Right & N/A \\
\hline $\mathrm{CF} 04$ & 64 & 160 & 60.3 & Right & N/A \\
\hline CM01 & 61 & 177.8 & 99.8 & Right & N/A \\
\hline CM02 & 64 & 182.9 & 86.2 & Right & N/A \\
\hline CM03 & 62 & 172.7 & 73.5 & Right & N/A \\
\hline CM04 & 61 & 175.3 & 70.3 & Right & N/A \\
\hline CM05 & 66 & 182.9 & 83.9 & Right & N/A \\
\hline $\begin{array}{l}\text { RCT mean } \\
\quad \pm \text { SD }\end{array}$ & $63.3 \pm 2.0$ & $169.8 \pm 11.0$ & $86.4 \pm 21.0$ & & \\
\hline $\begin{array}{c}\text { Control } \\
\text { mean } \pm \text { SD }\end{array}$ & $63.3 \pm 2.4$ & $171.6 \pm 9.5$ & $76.7 \pm 12.0$ & & \\
\hline \multicolumn{6}{|c|}{$\mathrm{R}$ : rotator cuff tear subject } \\
\hline \multicolumn{6}{|c|}{ C: control subject } \\
\hline \multicolumn{6}{|c|}{ F: female subject } \\
\hline \multicolumn{6}{|c|}{ M: male subject } \\
\hline N/A: not appl & cable & & & & \\
\hline
\end{tabular}

371 
373 Table 2: Functional tasks representative of activities of daily living. Participants were given

374 instructions on the start and target hand locations for each task, and were allowed to freely

375 choose their arm postures to reach the defined hand locations.

Task

\begin{tabular}{l}
\hline \hline Forward reach \\
\end{tabular}

Axilla wash ${ }^{2}$

Perineal care ${ }^{2}$

Hair comb ${ }^{2}$

${ }^{1}$ Subjects were seated at a table to perform the task

${ }^{2}$ Table was removed for task performance
Task instructions

Start with the humerus in $0^{\circ}$ elevation in the $90^{\circ}$ elevation plane (sagittal plane) and $2 \mathrm{lb}$ dumbbell resting on the table, reach with the weight a distance of $80 \%$ of forearm length, then return hand to starting position.

Start with humerus elevated in the $90^{\circ}$ elevation plane (sagittal plane) such that the hand reaches a distance of $80 \%$ of the subject's forearm length, holding the handle from a weight machine (6lb resistance), pull the handle until the humerus is in $0^{\circ}$ elevation in the $90^{\circ}$ elevation plane, then return hand to starting position.

Start with humerus in $0^{\circ}$ elevation in the $90^{\circ}$ elevation plane (sagittal plane) and 2lb dumbbell resting on the table, reach upward to set the weight on the shelf, then return hand to starting position.

Start with elbow extended and the humerus in $0^{\circ}$ elevation in the $90^{\circ}$ elevation plane (sagittal plane), resting quietly at the side, reach across the torso to touch the lateral aspect of the contralateral shoulder, then return hand to the starting position.

Start with elbow extended and the humerus in $0^{\circ}$ elevation in the $90^{\circ}$ elevation plane (sagittal plane), resting quietly at the side, reach behind the torso and touch the center of the small of the back with the palmar side of the fingers/hand, then return hand to the starting position.

Start with elbow extended and the humerus in $0^{\circ}$ elevation in the $90^{\circ}$ elevation plane (sagittal plane), resting quietly at the side holding a pencil (used to represent a comb), reach to the forehead, comb the center of the hair from front to back once and return hand to the starting position.
Load

$0.91 \mathrm{~kg}(2 \mathrm{lb})$ dumbbell (to mimic the weight of a typical kitchen object, like a plate)

$2.72 \mathrm{~kg}(6 \mathrm{lb})$ resistance from pulley system (to mimic the resistance associated with opening a door)

$0.91 \mathrm{~kg}(2 \mathrm{lb})$ dumbbell (to mimic the weight of a typical kitchen object, like a plate)

No load

No load

No load; subjects were given a pencil to mimic holding a comb 


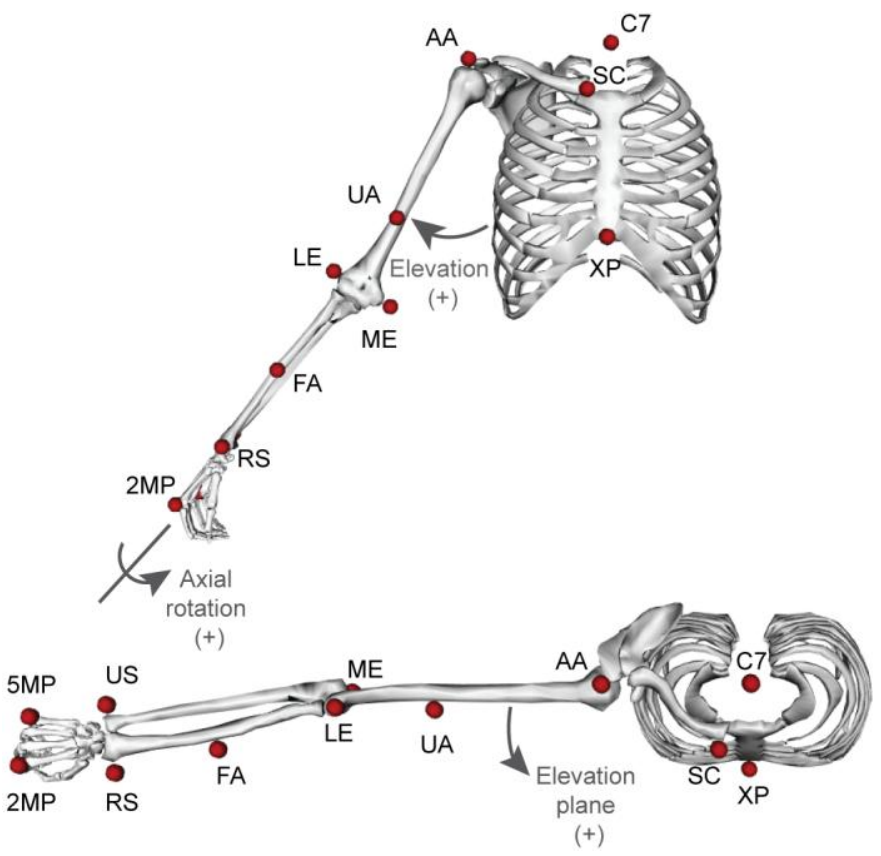

$379 \quad$ Figure 1 
A. Forward reach: elevation plane

$$
\begin{aligned}
& - \text { Control } \\
& -. \text { Rotator c } \\
& \hline
\end{aligned}
$$

B. Forward reach: axial rotation
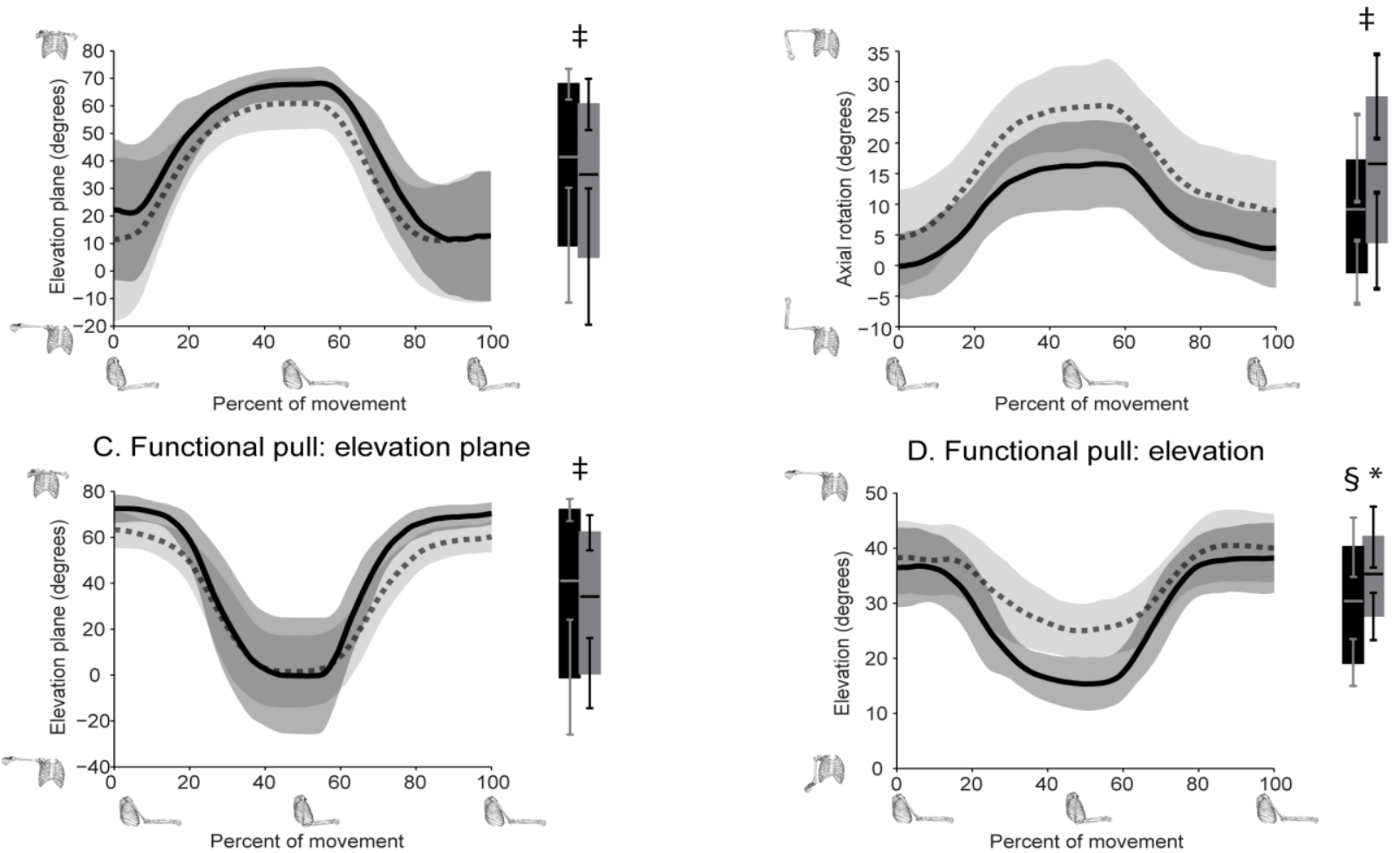

D. Functional pull: elevation

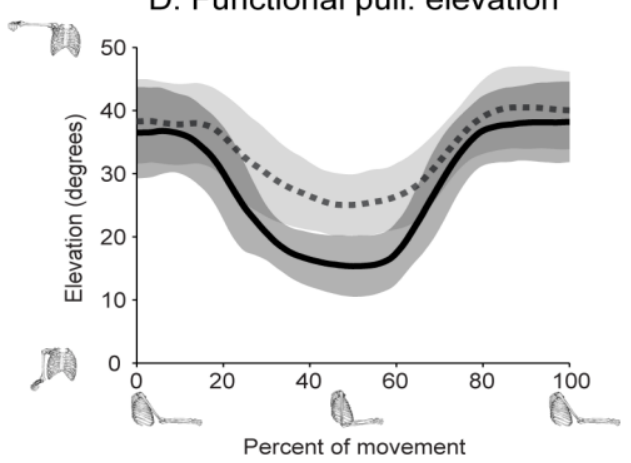

$\S *$
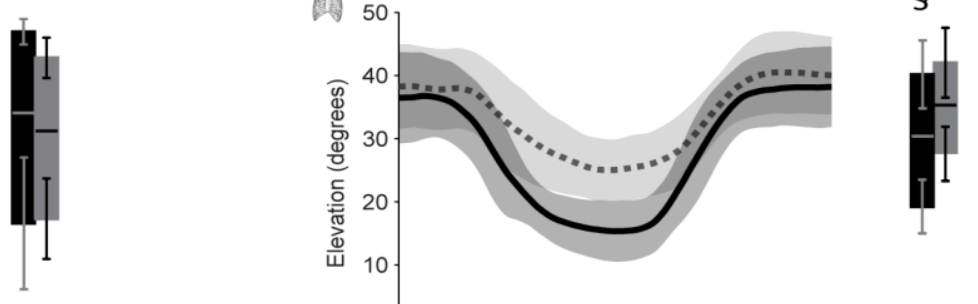

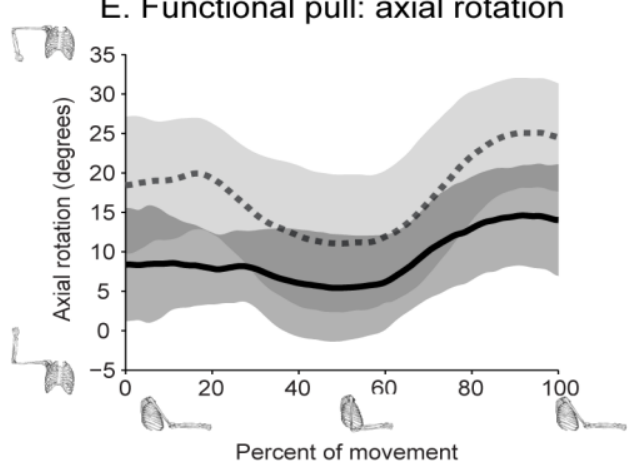

G. Axilla wash: elevation

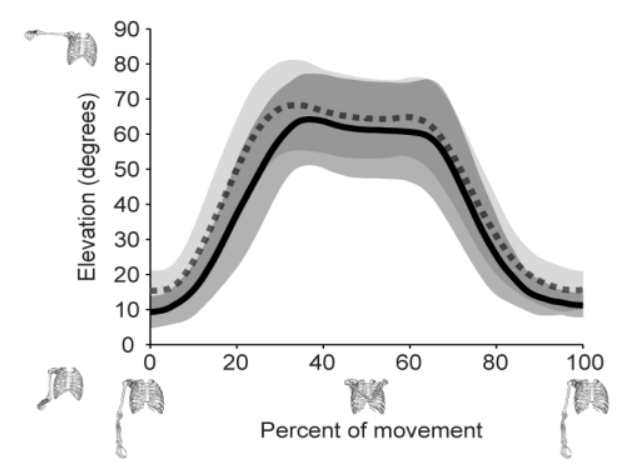

F. Upward reach $105^{\circ}$ : axial rotation

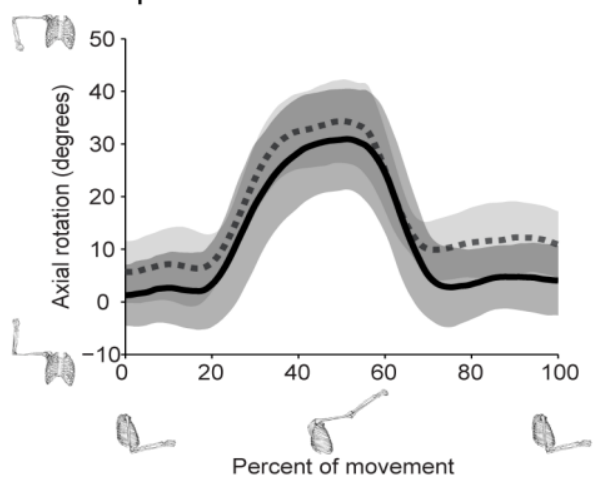

H. Hair comb: axial rotation

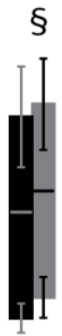

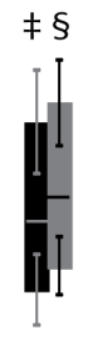

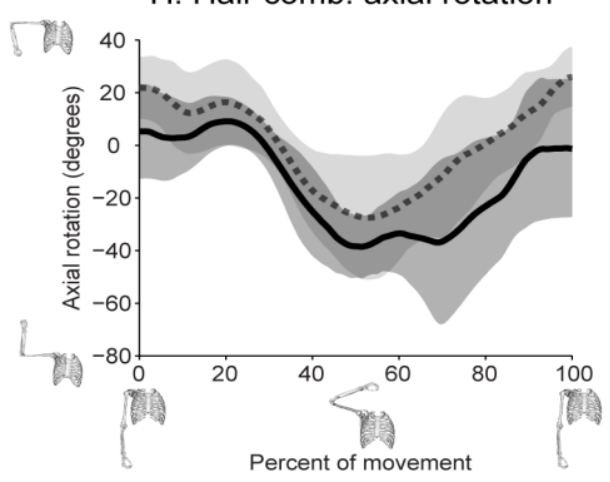

$\neq$

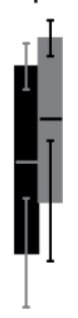

Figure 2 